\section{Validación de la escala de Borg en personas con diabetes mellitus tipo 2}

\author{
WALTER ROSALES ${ }^{1, \mathrm{a}}$, CRISTIAN COFRÉ CR,4, $^{3, \mathrm{~b}}$ \\ ALEJANDRA CICCHITTI ${ }^{2}$, CELINA BERTONA $^{2}$, \\ ALEJANDRO VIZCAYA², JOAQUÍN GONZÁLEZ ${ }^{2}$, \\ JORGE BAJUK ${ }^{2, c}$, MARTÍN RODRÍGUEZ ${ }^{2}$
}

\section{Validation of the Borg scale in participants with type 2 diabetes mellitus}

\begin{abstract}
Background: Borg Rating of Perceived Exertion Scale (RPE) is a psychophysiological measurements that translates physical stimuli to a psychological construct, which is perceived exertion. Its use is simple and it represents the effort perception. Each number of the scale is associated with a heart rate or percentage of maximal oxygen consumption ( $\mathrm{VO}_{2}$ max), which estimates the intensity of exercise. Aim: To validate RPE scale in patients with type 2 diabetes mellitus (DM2). Material and Methods: Thirty five DM2 participants with a median age of 59 years underwent an incremental exercise test in a treadmill with breath-by-breath analysis of oxygen consumption. After every three minute stage, the RPE scale was applied and correlated with oxygen consumption variables. Results: There was correlation coefficient of 0.91 between RPE score and $\mathrm{VO}_{2}$ max. A RPE score of 11 corresponded to $52 \%$ of $\mathrm{VO}_{2}$ max, a RPE score of 13 corresponded to $76 \%$ of $\mathrm{VO}_{2}$ max, a RPE of 15 corresponded to $88 \%$ of $\mathrm{VO}_{2}$ max and a RPE of 17 correspondent to a 100\% of $\mathrm{VO}_{2}$ max. Conclusions: $R P E$ Scale is valid in patients with type 2 diabetes as an estimate of the intensity of physical activity.
\end{abstract}

(Rev Med Chile 2016; 144: 1159-1163)

Key words: Diabetes Mellitus; Exercise; Physical Exertion; Rehabilitation.

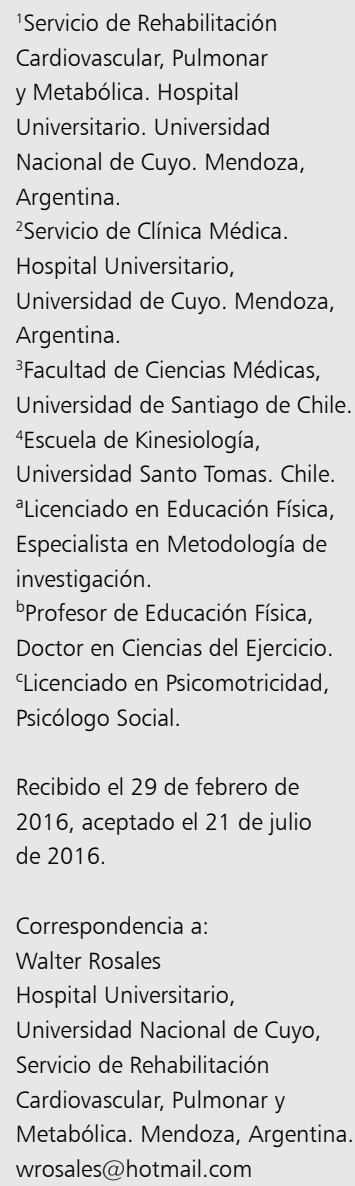

L a psicofísica es una rama de la psicología que estudia cómo un estímulo físico puede $\checkmark$ ser percibido por un individuo. Este campo no estaba suficientemente desarrollado hacia mediados de 1940, cuando el científico Gunnar Borg comenzó sus estudios con el objeto de contribuir al desarrollo de instrumentos válidos para utilizar lo sensorial como herramienta de medición de distintas variables, por ejemplo, para el control de la intensidad del ejercicio físico en distintos tipos de poblaciones ${ }^{1}$.

En el entrenamiento físico existen componentes que son fundamentales para poder administrar la carga de esfuerzo ${ }^{2}$, aspecto responsable de los beneficios biológicos para mejorar el rendimiento en un deporte determinado o para incrementar la calidad de vida en personas que padecen algún tipo de enfermedad, como las personas con diabetes mellitus de tipo 2 (DM2). En este contexto, el control de la intensidad del ejercicio es de vital importancia, y se hace fundamental hallar herramientas válidas, operativas y accesibles, para que los especialistas en ciencias del ejercicio puedan controlar esta variable.

El consumo de oxígeno es considerado el estándar de oro en la valoración de la potencia aeróbica y la capacidad funcional, esta variable fisiológica es fundamental, porque permite analizar sincrónicamente tres sistemas al mismo tiempo, el circulatorio, el respiratorio y el muscular ${ }^{3}$.

Existen distintas escalas de percepción del esfuerzo, para diferentes tipos de sintomatología, 
pero particularmente la escala de percepción del esfuerzo de Borg, que en inglés se denomina Rating of Perceived Exertion (RPE) ${ }^{4}$, es una de las más utilizadas, por haber demostrado un buen sustento científico ${ }^{5}$.

La RPE ha mostrado una correlación fuerte con variables como la frecuencia cardíaca ${ }^{6}$, esta última como expresión fisiológica del funcionamiento cardíaco es de mucha utilidad para controlar la intensidad de un esfuerzo aeróbico, pero su utilidad puede ser vulnerada por distintas situaciones, como la temperatura o fármacos betabloqueantes. Se considera necesaria una contrastación directa de esta escala con el estándar de oro ${ }^{7}$ en población diabética, con el fin de sumar al tratamiento del individuo diabético una herramienta que permita mejorar el control profesional e incluso el control autónomo de la terapia 8 .

La neuropatía diabética es una complicación crónica de la DM con posible influencia sobre las variables fisiológicas que afectan la percepción del esfuerzo. La fisiopatología de la neuropatía ${ }^{9}$ no es bien conocida, pero hay dos tesis que se postulan con fuerza de evidencia, una de ellas tiene que ver con hallazgos de alteracionaes metabólicas y vasculares. Por otro lado, la polineuropatía distal simétrica sensoriomotora es la tesis que prevalece, estudios clásicos ${ }^{10}$ han determinado la gran incidencia de este tipo de neuropatía, se ha observado que después de 25 años de padecer diabetes mellitus cerca de $50 \%$ de los pacientes la posee. Los nervios que se ven afectados en primer término son las porciones distales de los nervios más largos, por ello suelen aparecer primero las parestesias, entumecimiento, etc. de los dedos de los pies y manos antes que el resto de los miembros. Son alcanzadas con estas complicaciones degenerativas las grandes y pequeñas fibras nerviosas, mielinizadas o desmielinizadas, siendo su consecuencia clínica la dificultad para percibir el dolor, temperatura, o aparición de parestesias, disestesias, dolor visceral, disautonomía.

La percepción del esfuerzo, eje central de este estudio, radica en el supuesto de que el esfuerzo o cansancio percibido puede ser representado por medio de la escala de Borg durante el esfuerzo físico ${ }^{11}$, ya que la percepción del dolor muscular, la frecuencia respiratoria y otras variables, según Borg, se relacionan con esta escala.

Por otra parte, la neuropatía autonómica y su subclase, la neuropatía autonómica cardiovas- cular, es relevante para este trabajo debido a que esta última afecta el incremento de la frecuencia cardíaca, la ausencia o disminución de la variabilidad de la frecuencia cardíaca a los estímulos habituales; la hipotensión postural, la denervación sensitiva, la intolerancia al ejercicio físico, la disfunción ventricular izquierda y trastornos respiratorios ${ }^{12}$.

\section{Materiales y Métodos}

\section{Pacientes}

Se seleccionó una muestra de 35 pacientes con diabetes mellitus tipo 2, residentes en la ciudad de Mendoza, provincia de Mendoza, Argentina. El tipo de muestreo fue no probabilístico de casos consecutivos hasta alcanzar el número deseado. Se incluyeron en esta muestra aquellos que ${ }^{13}$ : 1) padecían diabetes mellitus tipo 2 (glucemia en ayunas $\geq 126 \mathrm{mg} / \mathrm{dl}$ o síntomas de hiperglucemia + glucemia casual $\geq 200 \mathrm{mg} / \mathrm{dl}$, o prueba de tolerancia a la glucosa (PTGO) $\geq 200 \mathrm{mg} / \mathrm{dl}$ después de 75 g de glucosa); 2) más de 30 años con diabetes tipo $2 ; 3)$ habían firmado el consentimiento informado para la realización de la ergometría con medición directa de consumo de oxígeno.

\section{Recolección de datos}

Luego de informar al paciente sobre las particularidades y riesgos de una prueba de esfuerzo cardiopulmonar o ergometría, se realizó a través del protocolo de Bruce Modificado ${ }^{14}$ en cinta de trote marca Kiprun KR-320/5 RS (Kip Machines, Argentina) y de un analizador de gases Cosmed Fitmate-PRO (COSMED, Argentina), con análisis directo de oxígeno respiración a respiración, el protocolo es de carácter máximo, en el cual fue incrementada la intensidad de la carga (velocidad e inclinación de la cinta deslizante) en etapas o estadios de tres minutos, en forma tal que al final de cada estadio de la prueba se pudo observar el comportamiento de distintas variables fisiológicas, como la actividad eléctrica del corazón, hemodinamia, análisis de gases $\left(\mathrm{VO}_{2}\right)$, y percepción del esfuerzo (RPE). Estos protocolos permiten que el organismo humano vaya adaptándose paulatinamente a una exigencia determinada, lo que favorece evaluar las variables antes mencionadas y en especial para este estudio, la correlación entre las variables. 


\section{Resguardos bioéticos}

El primer paso fue la invitación cursada a los potenciales participantes de la investigación. El consentimiento informado que se redactó para que cada paciente invitado diera su conformidad para participar de la investigación, se tomaron las precauciones y se consideraron las normas de Helsinki para intervención con seres humanos, además de las recomendaciones de la ley nacional de derechos del paciente 26.525/2009, resolución ANMAT 6.677/2010 y resolución del Ministerio de Salud de la Nación Argentina 1.480/2011 que aprueba la guía para investigaciones con seres humanos. Evaluado por el Comité de Ética del Hospital Universitario UNCuyo.

\section{Análisis estadístico}

Una vez realizadas las pruebas ergométricas, se aplicó el estadístico de Shapiro-Wilk para deter- minar si la muestra se ajustaba a una distribución normal o no y, de esta manera, determinar qué estadístico utilizar para establecer la correlación entre las variables centrales de este estudio. Posteriormente, se aplica la prueba de correlación de Pearson entre $\mathrm{VO}_{2} \%$ y RPE, debido a que la muestra se comportó con normalidad.

\section{Resultados}

La Tabla 1 muestra los datos relativos a las características de la muestra constituida por valores de media en: edad: 59,3 años; peso: 94,8; talla: 168,6; $\mathrm{VO}_{2}$ máximo: 25,3.

La Figura 1 presenta la relación entre la RPE y el $\mathrm{VO}_{2}$ máximo a través de la dispersión y la tendencia central de cada uno de los sujetos.

Los resultados obteniendos presentan una

Tabla 1. Se observa una descripción de las características de la muestra

\begin{tabular}{|lrrrrc|}
\hline Variables & n & Mínimo & Máximo & \multicolumn{1}{c|}{ Media } & Desv. típ. \\
\hline Sexo & 35 & 0,00 & 1,00 & 0,6300 & 0,48524 \\
Edad & 35 & 48,00 & 77,00 & 59,3300 & 7,86895 \\
Peso & 35 & 62,00 & 142,00 & 94,8140 & 18,62711 \\
Talla & 35 & 150,00 & 186,00 & 168,6100 & 9,61469 \\
VO$_{2}$ & 35 & 16,10 & 43,30 & 25,3560 & 5,78141 \\
N válido (según lista) & 35 & & & & \\
\hline
\end{tabular}

Variables: Sexo: 1 =masculino, 0=femenino; Edad: expresada en años; Peso: expresado en kilogramos, Talla=expresada en centímetros; $\mathrm{VO}_{2}$ : consumo de oxígeno máximo medido en forma directa.

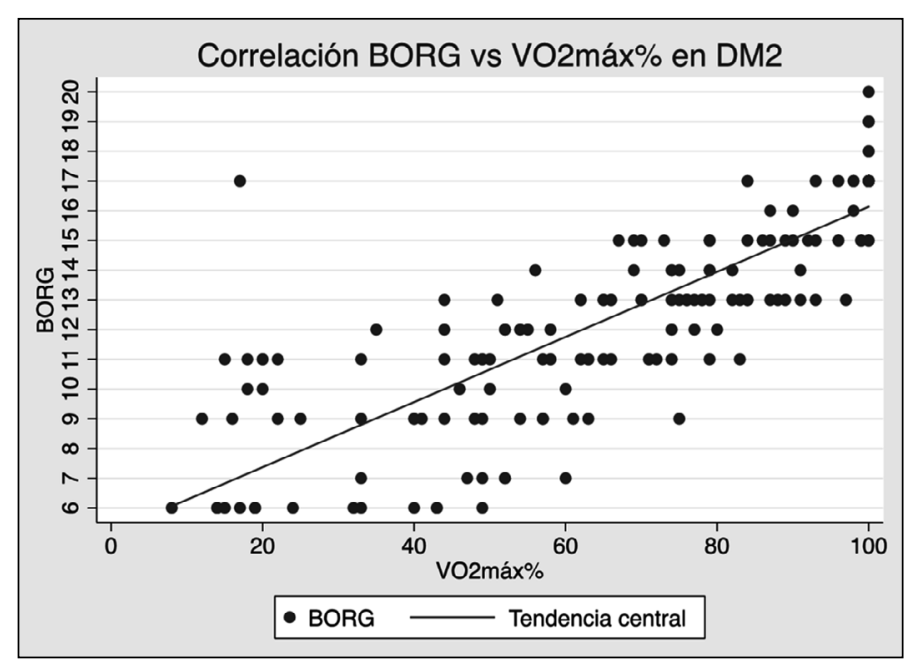

Figura 1. Borg vs $\mathrm{VO}_{2}$ máx\% en DM2. Dispersión y tendencia central. 
relación de la RPE 11 con 52\% del $\mathrm{VO}_{2}$ máx considerada intensidad moderada, RPE 13 asociada con $76 \%$ del $\mathrm{VO}_{2}$ máx determinada como una intensidad moderada-alta, RPE 15 con $88 \%$ del $\mathrm{VO}_{2}$ máx, considerada intensidad alta y RPE 17 con $100 \%$ del $\mathrm{VO}_{2}$ máx, marcando la máxima intensidad alcanzada.

\section{Discusión}

Los resultados del presente estudio han buscado validar el uso de la escala de Borg en pacientes diabéticos, se valora positivamente la utilidad de la RPE para contrastar los valores de los indicadores internos de intensidad obtenidos por medios directos, como ocurre en el caso de la FC, la que no siempre responde a la realidad del esfuerzo realizado, como lo mostraron varios trabajos ${ }^{15-18}$. Se entiende que existe un consenso en la literatura para establecer el uso de la RPE como medio de control de intensidad del ejercicio aeróbico, pero también durante la sesión de ejercicios en toda su globalidad, como lo mostraron algunas investigaciones ${ }^{16,19-22}$. La RPE ha sido usada como herramienta de control de la intensidad en varios trabajos científicos ${ }^{23}$, donde se utiliza la escala de Borg en pacientes con diabetes mellitus tipo 2 tal, como en nuestro estudio, es importante consignar que ya en el año 2000, el ACSM en su Position statement sobre ejercicio y diabetes tipo 2 , propone el control de la intensidad con escala de Borg ${ }^{24}$. La validación de la RPE ha sido realizada en diferentes grupos poblacionales, como en población infantil ${ }^{25}$, también en cardiópatas contrastada con saturación de oxígeno ${ }^{26}$, además de otros grupos como deportistas, sedentarios, etc. sin encontrar trabajos con las características particulares del presente estudio.

\section{Conclusión}

La Escala de Percepción del Esfuerzo de Borg resultó válida para su uso en pacientes con DM2 de más de 30 años de diagnóstico, como estimador de la intensidad del esfuerzo físico. Un valor RPE 11 se asoció con una intensidad moderada, RPE 13 moderada-alta, RPE 15 con intensidad alta y RPE 17 con máxima intensidad, al ser contrastada con el $\mathrm{VO}_{2}$. Esto permitirá a médicos y pacientes controlar con seguridad y efectividad este componente de la terapia física.

Agradecimientos: A Proyectos Basales y Vicerrectoria de Investigación, Desarrollo e Innovación de la Universidad de Santiago de Chile.

\section{Referencias}

1. Borg, G. Borg's Perceived Exertion and pain Scales. USA: Human Kinetics, 1998, p. 2.

2. Zamunér AR, Moreno MA, Camargo TM, Graetz JP, Rebelo ACS, Tamburús NY, da Silva E. Assessment of Subjective Perceived Exertion at the Anaerobic Threshold with the Borg Cr-10 Scale. J Sports Sci Med 2011; 10 (1): 130-6.

3. American College of Sports Medicine. Management for Persons With Chronic Diseases and Disabilities, Third Edition, United State of North America, Human Kinetics, 2009.

4. Mador MJ, Rodis A, Magalang UJ. Reproducibility of Borg scale measurements of dyspnea during exercise in patients with COPD. Chest 1995; 107 (6): 1590-7.

5. Borg G. Of the perception of speed when car-driving. Reports from the Department of Psychiatry, no 3. Umeå, Sweden: Umeå University. 1961.

6. Ekblom B, Goldberg A. The influence of physical training and other factors on the subjective rating of Perceived Exertion. USA: Acta Physiologica Scandinavica 83: 399-406.

7. Carmines E. Reliability and validity assessment, Serie Quantitative Applications in the Social Sciences, 17, Berverly Hills. Sage Publications. 1988.

8. Wasserman K. Exercise Testing and Interpretation, Fifth Edition, Philadelphia, Lippincott Williams \& Wilkins, 2012, p. 124-6.

9. Wackerhage H. Molecular Exercise Physiology: An introduction. First Edition, Routledge, Great Britain. 2014, p. 206-7.

10. Pirart J. Diabetes Mellitus and Its Degenerative Complications: A Prospective Study of 4400 Patients Observed Between 1947 to 1973. Diabete e Metabolisme 1973; 3 : 97-107.

11. Ehrman JK. Clinical Exercise Physiology, First Edition, United State of North America, Human Kinetics, 2003.

12. Romero Mestre JC. Neuropatía Autonómica Cardiovascular de la Diabetes Mellitus. Rev Cubana Endocrinol 1998; 9 (2): 149-63.

13. American Diabetes Association. Standards of Medical Care in Diabetes 2011.

14. American College of Sports Medicine. Manual ACSM 
para la valoración y prescripción del ejercicio (Vol. 44). Editorial Paidotribo, Barcelona. 2005.

15. Cortis C, Tessitore A, Lupo C, Pesce C, Fossile E, Figura F, et al. Inter-Limb Coordination, Strength, Jump, and Sprint Performances Following a Youth Men's Basketball Game. J Strength Cond Res 2011; 25 (1): 135-42.

16. Coutts AJ, Rampinini E, Marcora SM, Castagna C, Impellizzeri FM. Heart rate and blood lactate correlates of perceived exertion during small-sided soccer games. J Sci Med Sport 2009; 12 (1): 79-84.

17. Gómez-Piriz PT, Jiménez-Reyes P, Ruiz-Ruiz C. Relation Between Total Body Load and Session Rating of Perceived Exertion in Professional Soccer Players. J Strength Cond Res 2011; 25: 2100-3.

18. Impellizzeri FM, Rampinini E, Coutts AJ, Sassi A, Marcora SM. Use of RPE-based training load in soccer. Med Sci Sports Exerc 2004; 36 (6): 1042-7.

19. Manzi V, D’Ottavio S, Impellizzeri FM, Chaouachi A, Chamari K, Castagna C. Profile of weekly training load in elite male profesional basketball players. J Strength Cond Res 2010; 24 (5): 1399.

20. Milanez VF, Spiguel Lima MC, Gobatto CA, Perandini LA, Nakamura FY, Ribeiro LFP. Correlates of session-rate of perceived exertion (RPE) in a karate training session. Science \& Sports 2011; 26: 38-43.

21. Owen AL, Wong del P, McKenna M, Dellal A. Heart Rate Responses and Technical Comparison Between Small- vs. Large-Sided Games in Elite Professional Soccer. J Strength Cond Res 2011; 25: 2104-10.

22. Lockie RG, Murphy AJ, Scott BR, Janse de Jonge XA. Quantifying session ratings of perceived exertion for field-based speed training methods in team sport athletes. J Strength Cond Res 2012; 26 (10): 2721-8.

23. Murillo S, Novials A. Prescripción y tipo de ejercicio físico recomendado para pacientes con diabetes. Av Diabetol 2007; 23 (1): 33-9.

24. Albright A, Franz M, Hornsby G, Kriska A, Marrero D, Ullrich I, et al. American College of Sports Medicine position stand. Exercise and type 2 diabetes. Med Sci Sports Exerc 2000; 32 (7): 1345-60.

25. Núñez IR. Escala de medición de esfuerzo percibido infantil (EPInfant): validación en niños y adolescentes chilenos. Rev Chil Pediatr 2016; 87 (3): 211-2.

26. Valencia Chávez A, Jiménez Orozco JH, Díaz Marchán L, Mazadiego González ME. Correlación entre la escala de Borg modificada y la saturación de oxígeno durante la prueba de esfuerzo máxima en pacientes postinfartados. Rev Mex Med Fis Rehab 2012; 24 (1): 5-9. 